\title{
Meta-analysis of laparoscopic versus open liver resection for colorectal liver metastases
}

\author{
Zhi-qiang Tian ${ }^{1,2, *}$, Xiao-fang Su ${ }^{3, *}$, Zhi-yong Lin ${ }^{4, *}$, Meng-chao Wu ${ }^{1}$, Li-xin Wei ${ }^{1}$, \\ Jia $\mathrm{He}^{4}$ \\ ${ }^{1}$ Tumor Immunology and Gene Therapy Center, Eastern Hepatobiliary Surgery Hospital, The Second Military Medical \\ University, Shanghai 200438, China \\ ${ }^{2}$ Department of General Surgery, Wuxi People's Hospital Affiliated Nanjing Medical University, Wuxi, Jiangsu 214023, China \\ ${ }^{3}$ Department of Rehabilitation and Physiotherapy Medicine, Wuxi Taihu Hospital (101 Hospital of Chinese People's Liberation \\ Army), Wuxi, Jiangsu 214044, China \\ ${ }^{4}$ Department of Health Statistics, The Second Military Medical University, Shanghai 200433, China \\ *These authors have contributed equally to this work \\ Correspondence to: Li-xin Wei, email: weilixin_smmu@163.com \\ Jia He, email: hejia63@yeah.net
}

Keywords: colorectal liver metastases, laparoscopic liver resection, open liver resection, meta-analysis

Received: August 08, $2016 \quad$ Accepted: October 24, $2016 \quad$ Published: November 02, 2016

\section{ABSTRACT}

Background: To compare surgical and oncological outcomes of laparoscopic versus open liver resection for colorectal liver metastases.

Results: A total of 14 retrospective studies with 1679 colorectal liver metastases patients were analyzed: 683 patients treated with laparoscopic liver resection and 996 patients with open liver resection. With respect to surgical outcomes, laparoscopic compared with open liver resection was associated with lower blood loss (MD, -216.7, 95\% CI, -309.4 to $-124.1 ; P<0.00001$ ), less requiring blood transfusion (OR, 0.36; $95 \% \mathrm{CI}, 0.23$ to 0.55 ; $\mathrm{P}<0.00001$ ), lower postoperative complication morbidity (OR, 0.61; 95\% CI, 0.47 to $0.80 ; P=0.003$ ), and shorter hospitalization time (MD, $-3.85,95 \%$ CI, -5.00 to $-2.71 ; P<0.00001$ ). However, operation time and postoperative mortality were no significant difference between the two approaches. With respect to oncological outcomes, laparoscopic liver resection group was prone to lower recurrence rate $(\mathrm{OR}, 0.78 ; 95 \% \mathrm{CI}, 0.61-0.99 ; P=0.04)$, but surgical margins R0, overall survival and disease-free survival were no significant difference.

Materials and Methods: We performed a systematic search in MEDLINE, EMBASE, and CENTRAL for all relevant studies. All statistical analysis was performed using Review Manager version 5.3. Dichotomous data were calculated by odds ratio (OR) and continuous data were calculated by mean difference (MD) with $95 \%$ confidence intervals (CI).

Conclusions: Laparoscopic and open liver resection for colorectal liver metastases have the same effect on oncological outcomes, but laparoscopic liver resection achieves better surgical outcomes.

\section{INTRODUCTION}

Colorectal cancer is the third most common malignancies, and the liver is the most frequent site of metastasis [1,2]. Approximately $50 \%$ of colorectal cancer patients occur liver metastasis during disease evolution, which is a major cause of cancer death $[1,3]$. Liver resection is the only potential curative treatment for colorectal liver metastases with studies reporting 5-year survival rates of approximately $35-60 \%[3-7]$.
Laparoscopic surgery and open laparotomy are two surgical approaches of liver cancer resection. In recent years, laparoscopic liver resection is a growing option in the field of liver cancer surgery. Multiple studies [8-11] have attested to the effective in surgical outcomes and long-term oncologic outcomes of laparoscopic liver resection. However, no randomized controlled trial has been completed comparing laparoscopic with open liver resection for colorectal liver metastases [12]. Further, the related evidence of laparoscopic liver resection for 
colorectal liver metastases have not been systematically reviewed.

Although several meta-analyses [13-15] of observational studies have evaluated short- and long-term outcomes for laparoscopic liver resection, but there are limited data. It is necessary to carry out an updated metaanalysis in accordance to the Preferred Reporting Items for Systematic Reviews and Meta-Analyses (PRISMA) Statement [16] by pooling data from all of the available studies. Thus, we conduct a meta-analysis to identify and screen the benefits of laparoscopic compared with open liver resection for treatment colorectal liver metastases.

\section{RESULTS}

\section{Selected studies}

Our initial search strategy yielded 689 potential articles after removing duplicates in a combined search of MEDLINE (PubMed), EMBASE and CENTRAL databases covering studies published until October 18th 2016 and a manual approach. 637 articles were excluded on the basis of their titles and abstracts according to the inclusion and exclusion criteria. A full-text examination of the remaining 52 articles was performed. 38 additional articles were further ruled out for the reasons outlined in Figure 1. In the end, 14 studies published between 2002 and 2015 were included in quantitative synthesis in this meta-analysis. A flow diagram of the further details on selection process was shown in Figure 1.

\section{Study characteristics}

All included 14 studies [17-30] were retrospective studies that were conducted in Belgium (1), China (2), France (2), Japan (5), Norway (1), UK (1), and US (2). The informations about surgeons involved in these studies were not reported. 7 studies [17-19, 21, 25, 27, 28] had sample sizes greater than 100 patients. The baseline characteristics of these 14 studies in the meta-analysis were summarized in Table 1. A total of 1679 colorectal liver metastases patients were included in the meta-analysis: 683 patients treated with laparoscopic liver resection and 996 patients with open liver resection. All of studies stated that participators were consecutively enrolled.

\section{Quality judgments of studies}

The quality judgment for each study was performed with Newcastle-Ottawa Scale (NOS) [31]. The evaluation stars of each study based on NOS judgment were shown in Table 2. All studies reviewed consecutive colorectal liver metastases patients in the selection of patients. However, laparoscopic surgery was manipulated in selected patients who were suitable, and the patients accepting open surgery were selectively matched. All included studies were comparable. The mean value for the 14 retrospective cohort studies assessed was 6.9 stars. Overall, all of included studies were evaluated as being moderate to high quality.

\section{Meta-analysis of surgical outcomes}

With respect to surgical related outcomes, six endpoints including operation time, blood loss, perioperative blood transfusion, postoperative complication morbidity, postoperative mortality, and hospitalization time were taken into analysis. Dichotomous data was calculated by odds ratio (OR) and continuous data was calculated by mean difference (MD) with $95 \%$ confidence intervals (CI).

\section{Operation time}

The operation time was available from 10 studies $[17,18,20,21,23,25,26,28-30]$. Analysis indicated that there was low heterogeneity among the studies $(P=0.16$, $I^{2}=31 \%$ ) and a fixed effect model was used. Based on the complete analysis, operation time was assessed with no significant difference between laparoscopic and open liver resection for colorectal liver metastases (MD, 3.01; 95\% CI, -11.6 to $17.6 ; P=0.69$ ). (Figure 2 ).

\section{Blood loss}

Mean changes of blood loss were pooled for the 13 studies [17, 18, 20-30]. Heterogeneity was high $\left(P<0.00001, I^{2}=89 \%\right)$ and a random effect model was used. Intra-operative blood loss was significantly lower in laparoscopic liver resection than in open liver resection (MD, $-216.7,95 \%$ CI, -309.4 to $-124.1 ; P<0.00001$ ). (Figure 3).

\section{Perioperative blood transfusion}

The relative risk of perioperative blood transfusion was available form 7 studies [18-23, 25]. Analysis indicated that there was no heterogeneity among the studies $\left(P=0.47, I^{2}=0 \%\right)$ and a fixed effect model was used. The proportion of patients requiring blood transfusion was lower in laparoscopic liver resection than in open liver resection (OR, 0.36 ; $95 \%$ CI, 0.23 to 0.55 ; $P<0.00001$ ). (Figure 4).

\section{Postoperative complication morbidity}

All of 14 studies [17-30] reported on the postoperative complication morbidity rate. There was low significant heterogeneity among the studies $(P=0.25$, $I^{2}=18 \%$ ) and a fixed effect model was used. Patients in the laparoscopic liver resection had lower morbidity than in open liver resection (OR, $0.61 ; 95 \% \mathrm{CI}, 0.47$ to 0.80 ; $P=0.0003$ ). (Figure 5). 


\section{Postoperative mortality}

The relative risk of postoperative mortality was available for 6 studies $[17-19,21,27,28]$. There was no heterogeneity among the studies $\left(P=0.95, I^{2}=0 \%\right)$ and a fixed effect model was used. Perioperative mortality did not differ significantly between laparoscopic and open liver resection for colorectal liver metastases (OR, 0.48; $95 \% \mathrm{CI}, 0.15$ to $1.57 ; P=0.23$ ). (Figure 6 ).

\section{Hospitalization time}

The length of hospitalization time was pooled for all of 14 studies [17-30]. Heterogeneity was high among the studies $\left(P<0.0001, I^{2}=70 \%\right)$ and a random effect model was used. The pooled analysis showed that hospitalization time of laparoscopic liver resection was shorter than of open liver resection ( $\mathrm{MD},-3.85$ days, $95 \% \mathrm{CI},-5.00$ to $-2.71 ; P<0.00001$ ). (Figure 7).

\section{Meta-analysis of oncological outcomes}

With respect to oncological related outcomes, four endpoints including surgical margins R0, recurrence, disease-free survival, and overall survival were taken into analysis.

\section{Surgical margins R0}

The pathological resection margin status was reported in 11 studies $[17-19,21,23,25-30]$. Heterogeneity was low $\left(P=0.21, I^{2}=25 \%\right)$ and a fixed effect model was used. The pooled analysis showed that surgical margins R0 was assessed with difference

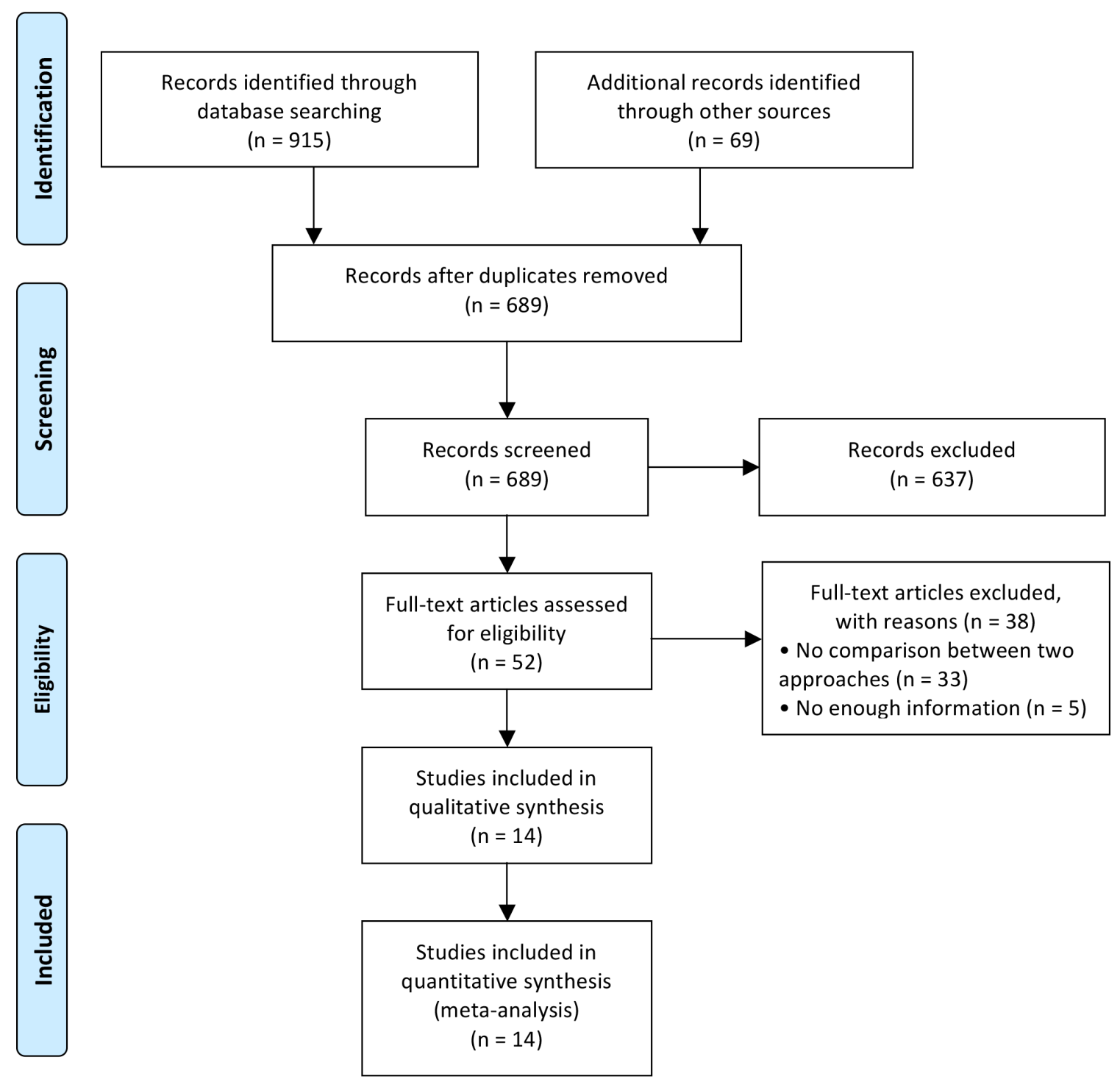

Figure 1: Flow diagram showing selection of relevant articles in the meta-analysis. 
Table 1: Baseline characteristics of the studies included in the meta-analysis

\begin{tabular}{|c|c|c|c|c|c|c|c|c|c|c|}
\hline Study (author, years, country) & Design & Arms & $\begin{array}{c}\text { No. of } \\
\text { patients }\end{array}$ & $\begin{array}{c}\text { Age } \\
\text { (years) }\end{array}$ & $\begin{array}{c}\text { Male } \\
(\%)\end{array}$ & $\begin{array}{l}\text { Tumor size } \\
\text { (cm) }\end{array}$ & $\begin{array}{c}\text { No. of } \\
\text { Tumors }\end{array}$ & $\begin{array}{c}\text { MH } \\
(N)\end{array}$ & $\begin{array}{c}\text { Conversion } \\
(n, \%)\end{array}$ & $\begin{array}{c}\text { Follow-up } \\
\text { (median, m) }\end{array}$ \\
\hline \multirow{2}{*}{ Abu et al., 2014, UK } & \multirow[t]{2}{*}{ retrospective } & LLR & 55 & $66(42-85)$ & 51 & $3.15(0.3-9)$ & $1(1-3)$ & 19 & $6(12)$ & 22 \\
\hline & & OLR & 85 & $67(47-86)$ & 65 & NA & NA & 46 & & 28 \\
\hline \multirow{2}{*}{ Bepuu et al., 2015, Japan } & \multirow{2}{*}{ PSM analysis } & LLR & 171 & $>70(n=61)$ & 63 & $>5(n=4)$ & $>2(n=44)$ & NR & NA & $>60^{\dagger}$ \\
\hline & & OLR & 342 & $>70(n=103)$ & 63 & $>5(n=8)$ & $>2(n=91)$ & NR & & $>60^{\dagger}$ \\
\hline \multirow{2}{*}{ Cannon et al., 2012, US } & \multirow[t]{2}{*}{ retrospective } & LLR & 35 & $62 \pm 10$ & NA & $4 \pm 3$ & $1 \pm 1$ & 19 & NA & $>60^{\dagger}$ \\
\hline & & OLR & 138 & $62 \pm 11$ & NA & $5 \pm 3$ & $1 \pm 1$ & 71 & & $>60^{\dagger}$ \\
\hline \multirow{2}{*}{ Castaing et al., 2009, France } & \multirow[t]{2}{*}{ retrospective } & LLR & 60 & $62 \pm 11$ & 62 & $3.3 \pm 1.1$ & $2.2 \pm 2.3$ & 26 & $6(10)$ & 30 \\
\hline & & OLR & 60 & $62 \pm 11$ & 62 & $4.4 \pm 3.8$ & $2.2 \pm 2.0$ & 24 & & 33 \\
\hline \multirow{2}{*}{ Cheung et al., 2012, China } & \multirow[t]{2}{*}{ retrospective } & LLR & 20 & $58(42-74)$ & 65 & $1.5(0.5-4.5)$ & $1(1-2)$ & 1 & 0 & NA \\
\hline & & OLR & 40 & $64(29-83)$ & 73 & $2.2(0.5-7)$ & $1(1-2)$ & 2 & & NA \\
\hline \multirow{2}{*}{ de'Angelis et al., 2015, France } & \multirow{2}{*}{ PSM analysis } & LLR & 52 & $63(32-81)$ & 52 & $2.6(1.5-6)$ & $1(1-4)$ & 18 & $3(5.8)$ & 59 \\
\hline & & OLR & 52 & $63(46-83)$ & 56 & $3.0(1.5-5.2)$ & $1(1-5)$ & 22 & & 54 \\
\hline \multirow{2}{*}{ Guerron et al., 2013, US } & \multirow[t]{2}{*}{ retrospective } & LLR & 40 & $66 \pm 1.9$ & 53 & $3.3 \pm 0.3$ & $1.3 \pm 0.1$ & 5 & $2(5)$ & 16 \\
\hline & & OLR & 40 & $62 \pm 1.8$ & 38 & $3.2 \pm 0.3$ & $1.7 \pm 0.1$ & 9 & & 16 \\
\hline \multirow{2}{*}{ Hasegawa et al., 2015, Japan } & \multirow[t]{2}{*}{ retrospective } & LLR & 100 & $67(24-91)$ & 64 & $2.3(0.7-9.5)$ & $1(1-8)$ & 20 & $1(1)$ & 29 \\
\hline & & OLR & 68 & $65(37-83)$ & 43 & $3.5(1.1-16)$ & $2(1-12)$ & 25 & & 36 \\
\hline \multirow{2}{*}{ Inoue et al., 2013, Japan } & \multirow[t]{2}{*}{ retrospective } & LLR & 23 & $66 \pm 9.6$ & 48 & $2.5 \pm 1.1$ & $\geq 2(n=0)$ & 4 & $1(4)$ & NA \\
\hline & & OLR & 24 & $68 \pm 9.5$ & 54 & $2.7 \pm 0.9$ & $\geq 2(n=3)$ & 5 & & NA \\
\hline \multirow{2}{*}{ Iwahashi et al., 2013, Japan } & \multirow[t]{2}{*}{ retrospective } & LLR & 21 & $68 \pm 11$ & 76 & $2.4 \pm 0.8$ & $1.9 \pm 1.1$ & 4 & NA & $>60^{\dagger}$ \\
\hline & & OLR & 21 & $68 \pm 10$ & 67 & $2.6 \pm 0.8$ & $2.1 \pm 1.2$ & 5 & & $>60^{\dagger}$ \\
\hline \multirow{2}{*}{ Kubota et al., 2014, Japan } & \multirow[t]{2}{*}{ retrospective } & LLR & 43 & $64 \pm 11$ & 51 & $\geq 5(n=8)$ & $\geq 2(n=16)$ & 7 & NA & 37 \\
\hline & & OLR & 62 & $66 \pm 12$ & 65 & $\geq 5(n=20)$ & $\geq 2(n=39)$ & 25 & & 37 \\
\hline \multirow{2}{*}{ Mala et al., 2002, Norway } & \multirow[t]{2}{*}{ retrospective } & LLR & 13 & $68(55-73)$ & 31 & $2.6(1-6)$ & $2(1-7)$ & 2 & 0 & NA \\
\hline & & OLR & 14 & $50(24-74)$ & 29 & $3(1.5-9)$ & $1(1-4)$ & 2 & & NA \\
\hline \multirow{2}{*}{ Qiu et al., 2013, China } & \multirow[t]{2}{*}{ retrospective } & LLR & 30 & $53 \pm 12$ & 47 & $2.5 \pm 2.0$ & $\geq 2(n=10)$ & 2 & $2(6.6)$ & NA \\
\hline & & OLR & 30 & NA & 50 & $2.8 \pm 1.5$ & $\geq 2(n=9)$ & 5 & & NA \\
\hline \multirow{2}{*}{ Topal et al., 2012, Belgium } & \multirow[t]{2}{*}{ retrospective } & LLR & 20 & 57.6 & 50 & $4(0.4-7)$ & $2(1-6)$ & 20 & NA & 43.4 \\
\hline & & OLR & 20 & 66.0 & 40 & $3.291-12.5$ & $2(1-14)$ & 20 & & 43.4 \\
\hline
\end{tabular}

PSM, propensity score matching; LLR, laparoscopic liver resection; OLR, open liver resection; MH, major hepatectomy ( $\geq 3$ segments of the liver); NA, not available; No., number; $\mathrm{m}$, month; $\uparrow$ upper ends of follow-up range.

in laparoscopic liver resection in comparison of OLR (OR, $1.50 ; 95 \%$ CI, 1.03 to $2.18 ; P=0.04)$. Open liver resection was prone to higher surgical margins R0, but the difference was slight. (Figure 8).

\section{Recurrence}

Concerning local recurrence, we pooled all hepatic and extra-hepatic recurrence of the included studies. 7 studies [17-19, 21, 22, 25, 28] were hit into our analysis with low heterogeneity $\left(P=0.29, I^{2}=18 \%\right)$ and a fixed effect model was used. Our outcome was prone to a lower recurrence rate in laparoscopic approach compared with open liver resection (OR, 0.78 ; 95\% CI, 0.61-0.99; $P=0.04$ ). (Figure 9).

\section{Disease-free survival}

The 5-year disease-free survival was available form 6 studies [18, 19, 21, 24, 27, 28]. Heterogeneity was high $\left(P=0.06, I^{2}=52 \%\right)$ and a random effect model was used. The 5-year disease-free survival was no significant difference between laparoscopic and open liver resection for colorectal liver metastases (OR, $0.88 ; 95 \% \mathrm{CI}, 0.53$ to $1.47 ; P=0.63$ ). (Figure 10 ).

\section{Overall survival}

There were 6 studies [18, 19, 21, 24, 27, 28] reported the 5-year overall survival, which was the crucial endpoint. Heterogeneity was high $(P=0.003$, $\left.I^{2}=72 \%\right)$ and a random effect model was used. The findings indicated that the 5-year overall survival was no significant difference between laparoscopic and open liver resection for colorectal liver metastases (OR, 0.88 ; 95\% $\mathrm{CI}, 0.49$ to $1.58 ; P=0.68$ ). (Figure 11 ).

\section{Publication bias}

The funnel plot of postoperative complication morbidity were assessed to evaluate the reliability of publication bias in this meta-analysis [32]. The funnel plot 
Table 2: Quality assessment of studies in the meta-analysis based on newcastle-ottawa scale

\begin{tabular}{|c|c|c|c|c|c|c|c|c|c|}
\hline \multirow{2}{*}{ Study (Author, years) } & \multicolumn{4}{|c|}{ Selection } & \multirow{2}{*}{$\frac{\text { Comparability }}{1}$} & \multicolumn{3}{|c|}{ Outcome } & \multirow[t]{2}{*}{ Quality judgment } \\
\hline & 1 & 2 & 3 & 4 & & 1 & 2 & 3 & \\
\hline Abu et al., 2014 & $\star$ & - & $\star$ & $\star$ & $\star$ & $\star$ & - & $\star$ & 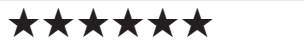 \\
\hline Bepuu et al., 2015 & $\star$ & - & $\star$ & $\star$ & $\star \star \star$ & $\star$ & $\star$ & $\star$ & 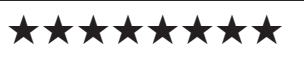 \\
\hline Cannon et al., 2012 & $\star$ & $\star$ & - & - & $\star \star$ & $\star$ & $\star$ & $\star$ & 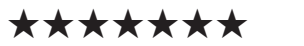 \\
\hline Castaing et al., 2009 & $\star$ & $\star$ & $\star$ & - & $\star \star$ & $\star$ & - & $\star$ & 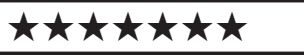 \\
\hline Cheung et al., 2012 & $\star$ & $\star$ & $\star$ & $\star \star$ & $\star$ & $\star$ & - & $\star$ & 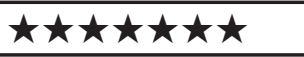 \\
\hline de'Angelis et al., 2015 & $\star$ & - & $\star$ & - & $\star \star$ & $\star$ & $\star$ & $\star$ & 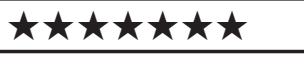 \\
\hline Guerron et al., 2013 & $\star$ & $\star$ & $\star$ & $\star$ & $\star$ & $\star$ & - & $\star$ & 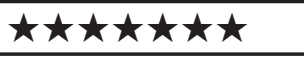 \\
\hline Hasegawa et al., 2015 & $\star$ & $\star$ & $\star$ & - & $\star \star$ & $\star$ & $\star$ & $\star$ & 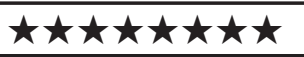 \\
\hline Inoue et al., 2013 & $\star$ & - & $\star$ & - & $\star \star \star$ & $\star$ & - & $\star$ & 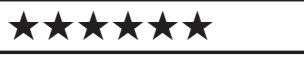 \\
\hline Iwahashi et al., 2013 & $\star$ & $\star$ & - & - & $\star \star \star$ & $\star$ & $\star$ & $\star$ & 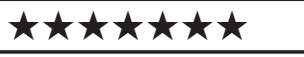 \\
\hline Kubota et al., 2014 & $\star$ & $\star$ & $\star$ & - & $\star$ & $\star$ & $\star$ & $\star$ & 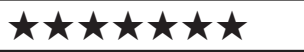 \\
\hline Mala et al., 2002 & $\star$ & - & - & - & $\star \star \star$ & $\star$ & - & $\star$ & 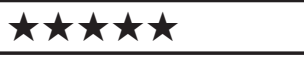 \\
\hline Qiu et al., 2013 & $\star$ & $\star$ & $\star$ & $\star$ & $\star \star$ & $\star$ & - & $\star$ & 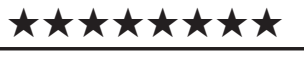 \\
\hline Topal et al., 2012 & $\star$ & $\star$ & - & - & $\star \star \star$ & $\star$ & - & $\star$ & 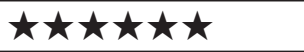 \\
\hline
\end{tabular}

was basically inverted and funnel-shaped with bilateral symmetry, indicating that there was no publication bias. (Figure 12).

\section{DISCUSSION}

Laparoscopic liver resection for colorectal liver metastases has not been widely accepted [15]. The mainly reason is lack of convincing evidence in adequacy comparison of surgical outcomes and oncological value [14]. To date, numerous retrospective and comparative studies [17-30] have reported the feasibility, safety, and efficacy of laparoscopic liver resection for colorectal liver metastases. However, no randomized controlled trial has been completed on laparoscopic compared with open liver resection for colorectal liver metastases for ethical and other reasons. As far as we know, one randomized controlled trial (the Oslo-CoMet Study, NCT01516710) [12] is in progress comparing laparoscopic with open liver resection for colorectal liver metastases, but the long-term result will not be available before 2020 . Therefore, non-randomized studies may thus be useful by providing information on treatment effects. Our goal of this meta-analysis was to evaluate the surgical and oncological outcomes of laparoscopic compared with open liver resection for colorectal liver metastases. So, we comprehensively identified all relevant non-randomized studies and summarized the evidence on surgical and oncological outcomes.

With respect to surgical outcomes, postoperative complication morbidity is a major cause of patient suffering and societal costs. Our analysis demonstrates

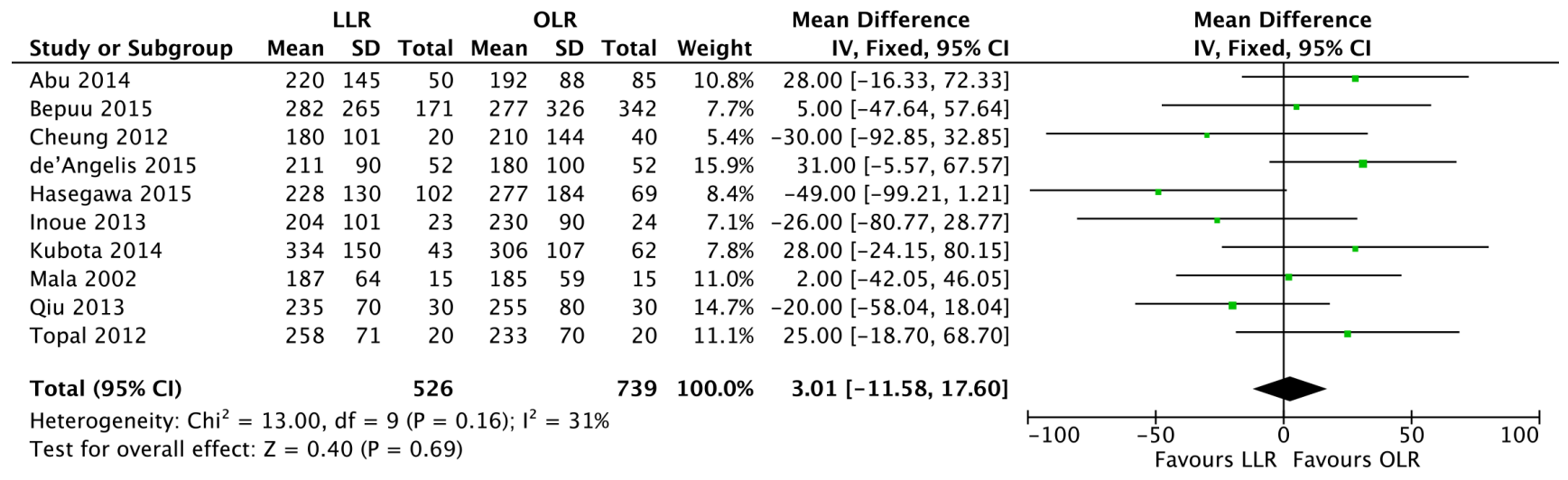

Figure 2: Forest plot displaying operation time (min) of the meta-analysis. LLR, laparoscopic liver resection; OLR, open liver resection. 


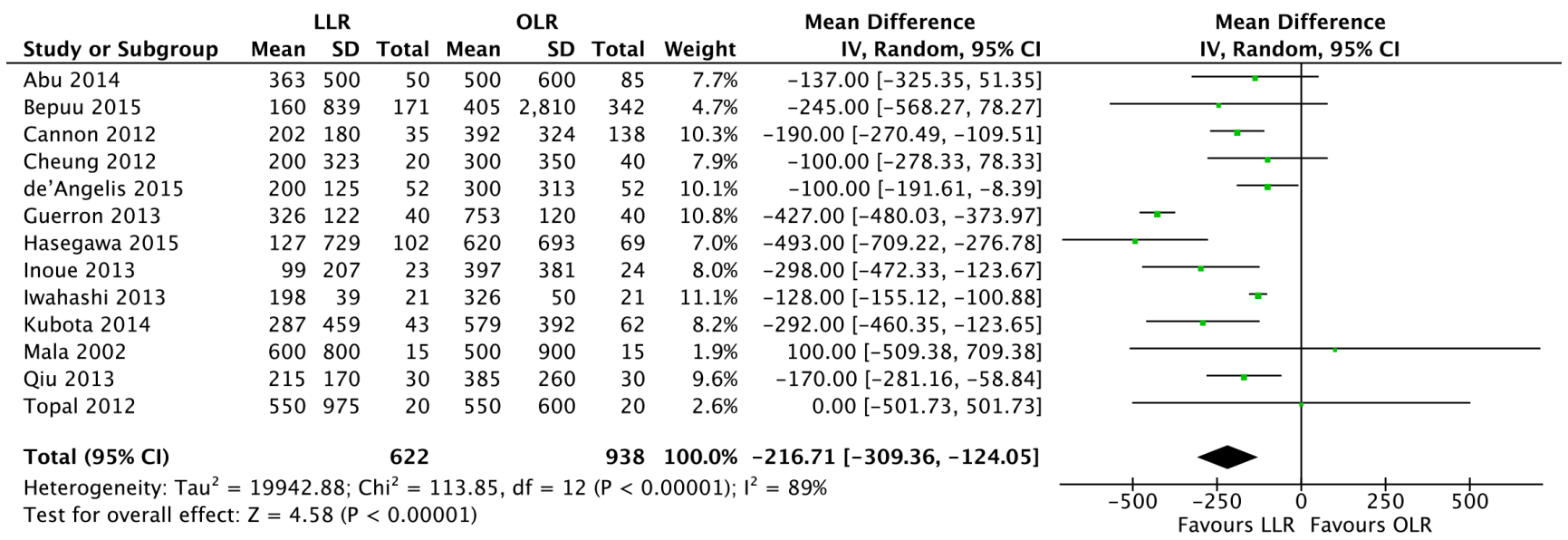

Figure 3: Forest plot displaying blood loss (ml) of the meta-analysis. LLR, laparoscopic liver resection; OLR, open liver resection.

\begin{tabular}{|c|c|c|c|c|c|c|c|c|c|c|}
\hline Study or Subgroup & \multicolumn{2}{|l|}{ LLR } & \multicolumn{2}{|c|}{ OLR } & \multicolumn{3}{|c|}{ Odds Ratio } & \multicolumn{2}{|c|}{$\begin{array}{c}\text { Odds Ratio } \\
\text { M-H, Fixed, } 95 \% \mathrm{Cl}\end{array}$} & \\
\hline Bepuu 2015 & 14 & 171 & 44 & 342 & $34.2 \%$ & $0.60[0.32,1.14]$ & & $\longrightarrow$ & & \\
\hline Castaing 2009 & 9 & 60 & 22 & 60 & $23.8 \%$ & $0.30[0.13,0.74]$ & & & & \\
\hline Cheung 2012 & 0 & 20 & 1 & 40 & $1.3 \%$ & $0.64[0.03,16.48]$ & & & & \\
\hline de'Angelis 2015 & 3 & 52 & 11 & 52 & $13.2 \%$ & $0.23[0.06,0.87]$ & & & & \\
\hline Guerron 2013 & 2 & 40 & 8 & 40 & $9.7 \%$ & $0.21[0.04,1.06]$ & & & & \\
\hline Inoue 2013 & 1 & 23 & 4 & 24 & $4.8 \%$ & $0.23[0.02,2.21]$ & & & & \\
\hline Kubota 2014 & 1 & 43 & 13 & 62 & $13.2 \%$ & $0.09[0.01,0.71]$ & & & & \\
\hline Total $(95 \% \mathrm{Cl})$ & & 409 & & 620 & $100.0 \%$ & $0.36[0.23,0.55]$ & & & & \\
\hline Total events & 30 & & 103 & & & & & & & \\
\hline $\begin{array}{l}\text { Heterogeneity: } \mathrm{Chi}^{2}= \\
\text { Test for overall effect }\end{array}$ & $\begin{array}{l}5.58, \mathrm{df} \\
Z=4.64\end{array}$ & $\begin{array}{l}=6(\mathrm{P} \\
+(\mathrm{P}<\mathrm{C}\end{array}$ & $\begin{array}{l}=0.47) ; 1 \\
.00001)\end{array}$ & $1^{2}=0 \%$ & & & 0.01 & 0.1 Favours LLR & $\begin{array}{r}10 \\
\text { Favours OLR }\end{array}$ & 100 \\
\hline
\end{tabular}

Figure 4: Forest plot displaying perioperative blood transfusion of the meta-analysis. LLR, laparoscopic liver resection; OLR, open liver resection.

\begin{tabular}{|c|c|c|c|c|c|c|c|c|c|c|}
\hline Study or Subgroup & $\begin{array}{c}\text { LLR } \\
\text { Events } \\
\end{array}$ & Total & $\begin{array}{c}\text { OLR } \\
\text { Events } \\
\end{array}$ & Total & Weight & $\begin{array}{c}\text { Odds Ratio } \\
\text { M-H, Fixed, 95\% Cl }\end{array}$ & \multicolumn{4}{|c|}{$\begin{array}{c}\text { Odds Ratio } \\
\text { M-H, Fixed, 95\% Cl }\end{array}$} \\
\hline Abu 2014 & 8 & 50 & 24 & 85 & $10.5 \%$ & $0.48[0.20,1.18]$ & & & & \\
\hline Bepuu 2015 & 24 & 171 & 44 & 342 & $17.8 \%$ & $1.11[0.65,1.89]$ & & & & \\
\hline Cannon 2012 & 8 & 35 & 67 & 138 & $14.7 \%$ & $0.31[0.13,0.74]$ & & & & \\
\hline Castaing 2009 & 16 & 60 & 17 & 60 & $8.8 \%$ & $0.92[0.41,2.05]$ & & & & \\
\hline Cheung 2012 & 2 & 20 & 2 & 40 & $0.8 \%$ & $2.11[0.27,16.21]$ & & & & \\
\hline de'Angelis 2015 & 9 & 52 & 11 & 52 & $6.4 \%$ & $0.78[0.29,2.08]$ & & & & \\
\hline Guerron 2013 & 6 & 40 & 8 & 40 & $4.8 \%$ & $0.71[0.22,2.26]$ & & & & \\
\hline Hasegawa 2015 & 9 & 102 & 17 & 69 & $13.0 \%$ & $0.30[0.12,0.71]$ & & & & \\
\hline Inoue 2013 & 2 & 23 & 5 & 24 & $3.1 \%$ & $0.36[0.06,2.09]$ & & & & \\
\hline Iwahashi 2013 & 2 & 21 & 5 & 21 & $3.2 \%$ & $0.34[0.06,1.98]$ & & & & \\
\hline Kubota 2014 & 1 & 43 & 6 & 62 & $3.4 \%$ & $0.22[0.03,1.92]$ & & & & \\
\hline Mala 2002 & 2 & 15 & 4 & 14 & $2.5 \%$ & $0.38[0.06,2.54]$ & & & & \\
\hline Qiu 2013 & 8 & 30 & 15 & 30 & $7.7 \%$ & $0.36[0.12,1.07]$ & & & & \\
\hline Topal 2012 & 7 & 20 & 7 & 20 & $3.2 \%$ & $1.00[0.27,3.67]$ & & & & \\
\hline Total $(95 \% \mathrm{Cl})$ & & 682 & & 997 & $100.0 \%$ & $0.61[0.47,0.80]$ & & & & \\
\hline Total events & 104 & & 232 & & & & & & & \\
\hline $\begin{array}{l}\text { Heterogeneity: } \mathrm{Chi}^{2} \\
\text { Test for overall effec }\end{array}$ & $\begin{array}{l}15.93, d f \\
Z=3.59\end{array}$ & $\begin{array}{l}f=13 \\
(P=0\end{array}$ & $\begin{array}{l}(P=0.25 \\
.0003)\end{array}$ & ); $1^{2}=$ & & & 0.02 & 0.1 Favours LLR & $\begin{array}{rr}10 \\
\text { Favours OLR }\end{array}$ & 50 \\
\hline
\end{tabular}

Figure 5: Forest plot displaying postoperative complication morbidity of the meta-analysis. LLR, laparoscopic liver resection; OLR, open liver resection. 


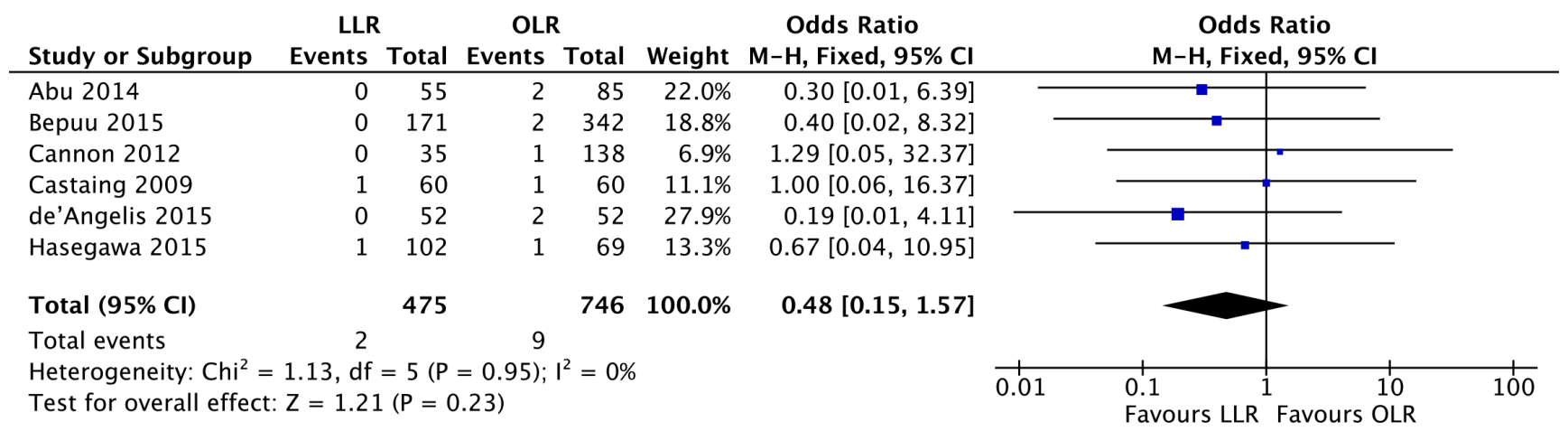

Figure 6: Forest plot displaying postoperative mortality of the meta-analysis. LLR, laparoscopic liver resection; OLR, open liver resection.

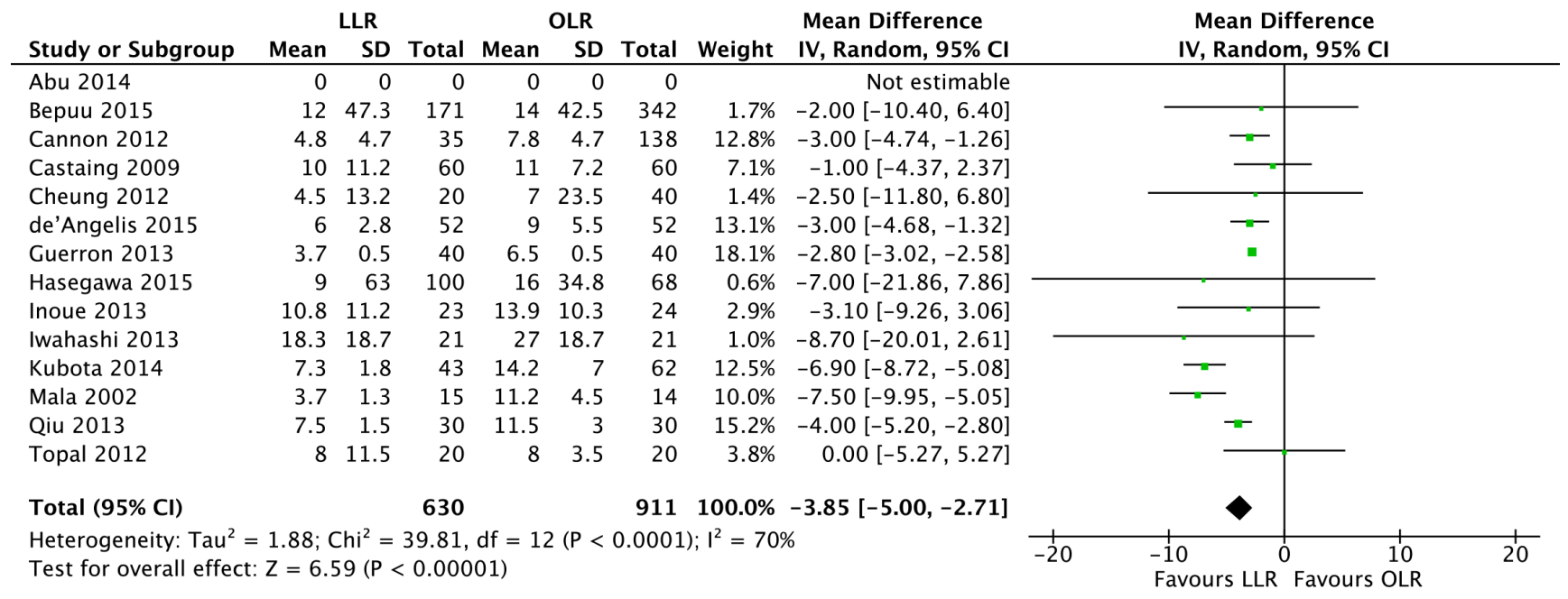

Figure 7: Forest plot displaying hospitalization time (days) of the meta-analysis. LLR, laparoscopic liver resection; OLR, open liver resection.

\begin{tabular}{|c|c|c|c|c|c|c|c|c|c|c|}
\hline Study or Subgroup & $\begin{array}{c}\text { LLR } \\
\text { Events } \\
\end{array}$ & LLR & \multicolumn{2}{|l|}{ OLR } & Weight & $\begin{array}{c}\text { Odds Ratio } \\
\text { M-H, Fixed, } 95 \% \mathrm{Cl}\end{array}$ & & \multicolumn{2}{|c|}{$\begin{array}{c}\text { Odds Ratio } \\
\text { M-H, Fixed, } 95 \% \mathrm{Cl}\end{array}$} & \\
\hline Abu 2014 & 48 & 50 & 80 & 85 & $5.1 \%$ & $1.50[0.28,8.04]$ & & & & \\
\hline Bepuu 2015 & 154 & 171 & 315 & 342 & $44.9 \%$ & $0.78[0.41,1.47]$ & & & & \\
\hline Cannon 2012 & 35 & 35 & 112 & 138 & $1.4 \%$ & $16.72[0.99,281.47]$ & & & & \\
\hline Castaing 2009 & 52 & 60 & 43 & 60 & $12.3 \%$ & $2.57[1.01,6.53]$ & & & & \\
\hline de'Angelis 2015 & 43 & 52 & 46 & 52 & $17.1 \%$ & $0.62[0.20,1.90]$ & & & & \\
\hline Hasegawa 2015 & 95 & 102 & 63 & 69 & $11.1 \%$ & $1.29[0.42,4.03]$ & & & & \\
\hline Inoue 2013 & 22 & 23 & 21 & 24 & $1.9 \%$ & $3.14[0.30,32.65]$ & & & & \\
\hline Kubota 2014 & 43 & 43 & 58 & 62 & $1.2 \%$ & $6.69[0.35,127.60]$ & & & & \\
\hline Mala 2002 & 12 & 13 & 12 & 14 & $1.9 \%$ & $2.00[0.16,25.11]$ & & & & \\
\hline Qiu 2013 & 30 & 30 & 27 & 30 & $1.0 \%$ & $7.76[0.38,157.14]$ & & & & \\
\hline Topal 2012 & 19 & 20 & 19 & 20 & $2.0 \%$ & $1.00[0.06,17.18]$ & & & & \\
\hline Total $(95 \% \mathrm{Cl})$ & & 599 & & 896 & $100.0 \%$ & $1.50[1.03,2.18]$ & & & & \\
\hline Total events & 553 & & 796 & & & & & & & \\
\hline $\begin{array}{l}\text { Heterogeneity: } \mathrm{Chi}^{2} \\
\text { Test for overall effec }\end{array}$ & $\begin{array}{l}13.28, d \\
Z=2.11\end{array}$ & $\begin{array}{l}\text { If }=10 \\
1(P=0\end{array}$ & $\begin{array}{l}(P=0.21 \\
0.04)\end{array}$ & $1^{2}=$ & & & 0.005 & $\begin{array}{c}0.1 \\
\text { Favours LLR }\end{array}$ & $\begin{array}{c}10 \\
\text { Favours OLR }\end{array}$ & 200 \\
\hline
\end{tabular}

Figure 8: Forest plot displaying surgical margins R0 of the meta-analysis. LLR, laparoscopic liver resection; OLR, open liver resection. 
that laparoscopic liver resection acquired lower postoperative morbidity than open liver resection. Similar finding is also reported by multiple observational studies, in which morbidity rate for laparoscopic liver resection varies from $11 \%$ to $15 \%$ [33-35] and for open liver resection varies from $22 \%$ to $47 \%[36,37]$. In terms of

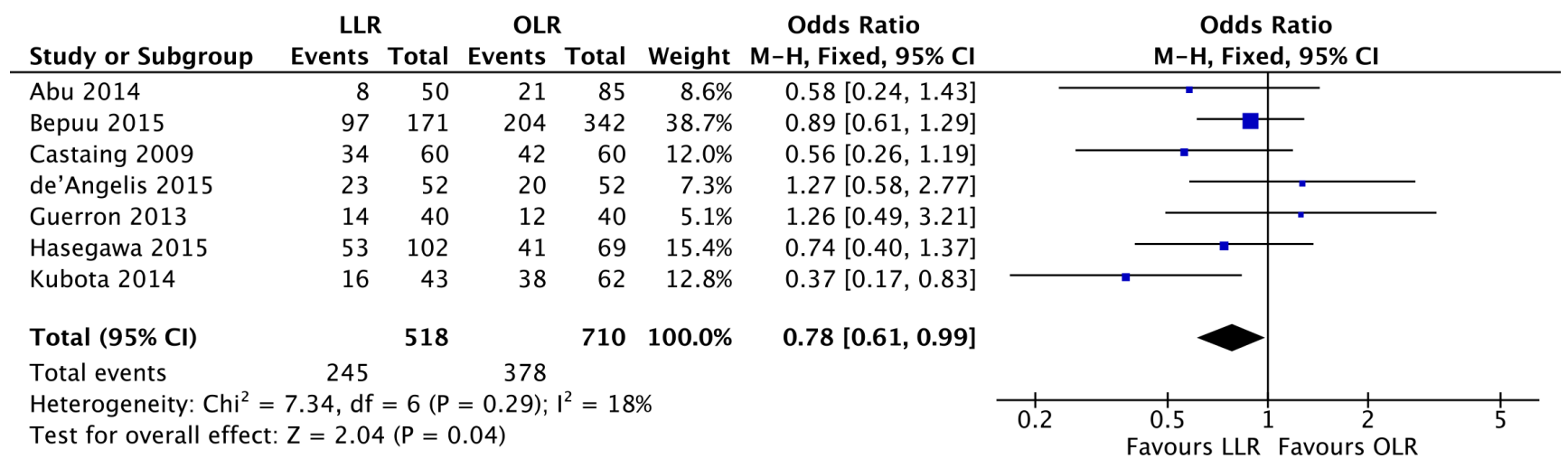

Figure 9: Forest plot displaying recurrence of the meta-analysis. LLR, laparoscopic liver resection; OLR, open liver resection.

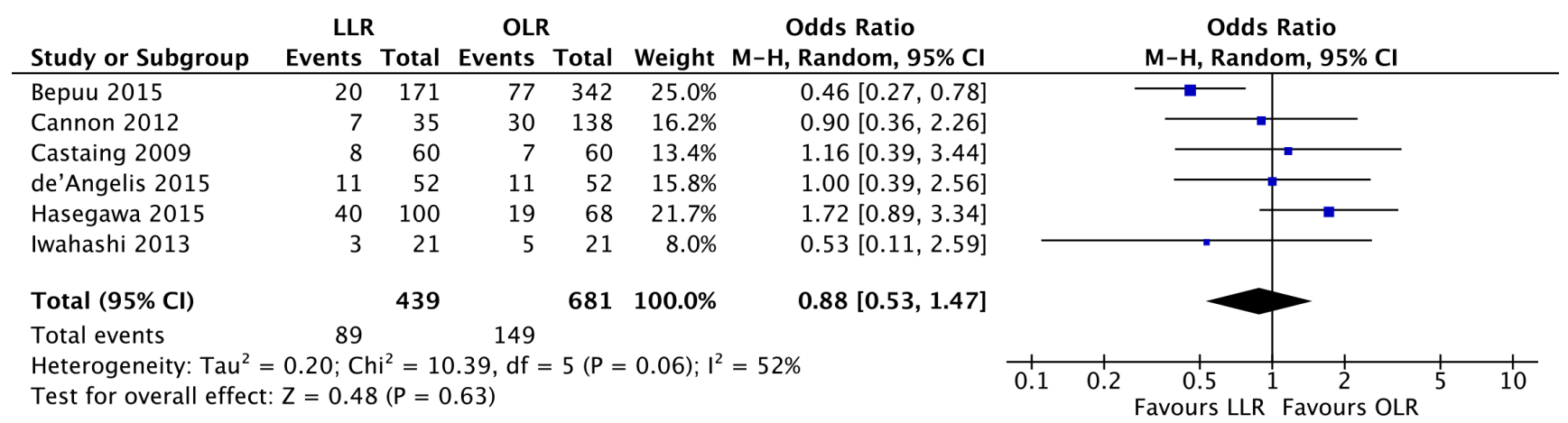

Figure 10: Forest plot displaying disease-free survival of the meta-analysis. LLR, laparoscopic liver resection; OLR, open liver resection.

\begin{tabular}{|c|c|c|c|c|c|c|c|c|c|c|}
\hline Study or Subgroup & LLR & Total & $\begin{array}{c}\text { OLR } \\
\text { Events } \\
\end{array}$ & Total & Weight & $\begin{array}{c}\text { Odds Ratio } \\
\text { M-H, Random, } 95 \% \mathrm{Cl}\end{array}$ & & \multicolumn{2}{|c|}{$\begin{array}{c}\text { Odds Ratio } \\
\mathrm{M}-\mathrm{H}, \text { Random, } 95 \% \mathrm{Cl}\end{array}$} & \\
\hline Bepuu 2015 & 29 & 171 & 124 & 342 & $21.2 \%$ & $0.36[0.23,0.57]$ & & $\longrightarrow$ & & \\
\hline Cannon 2012 & 13 & 35 & 51 & 138 & $17.1 \%$ & $1.01[0.47,2.17]$ & & & & \\
\hline Castaing 2009 & 10 & 60 & 10 & 60 & $14.6 \%$ & $1.00[0.38,2.61]$ & & & & \\
\hline de'Angelis 2015 & 38 & 52 & 33 & 52 & $16.2 \%$ & $1.56[0.68,3.60]$ & & & & \\
\hline Hasegawa 2015 & 57 & 100 & 33 & 68 & $19.1 \%$ & $1.41[0.76,2.61]$ & & & & \\
\hline Iwahashi 2013 & 9 & 21 & 11 & 21 & $11.8 \%$ & $0.68[0.20,2.30]$ & & & & \\
\hline Total $(95 \% \mathrm{Cl})$ & & 439 & & 681 & $100.0 \%$ & $0.88[0.49,1.58]$ & & & & \\
\hline Total events & 156 & & 262 & & & & & & & \\
\hline $\begin{array}{l}\text { Heterogeneity: } \mathrm{Tau}^{2} \\
\text { Test for overall effec }\end{array}$ & $\begin{array}{l}0.36 ; C h \\
Z=0.42\end{array}$ & $\begin{array}{l}\mathrm{i}^{2}=17 \\
(\mathrm{P}=0\end{array}$ & $\begin{array}{l}.94, \mathrm{df}= \\
.68)\end{array}$ & $=5(P=$ & $=0.003)$ & $1^{2}=72 \%$ & 0.05 & $\begin{array}{l}0.2 \quad 1 \\
\text { Favours LLR }\end{array}$ & \begin{tabular}{|c} 
\\
\end{tabular} & 20 \\
\hline
\end{tabular}

Figure 11: Forest plot displaying overall survival of the meta-analysis. LLR, laparoscopic liver resection; OLR, open liver resection. 
the other surgical outcomes, our analysis showed that colorectal liver metastases patients with laparoscopic liver resection acquired lower blood loss, less blood transfusion requirements and shorter hospitalization time than with open liver resection. However, postoperative mortality was no significantly different between the two approaches. This meta-analysis demonstrates that laparoscopic liver resection for colorectal liver metastases achieves better surgical outcomes than open liver resection.

The oncological outcomes are the core indicators for resection treatment of colorectal liver metastases. Specific concerns about the oncologic adequacy of laparoscopic liver resections include port site metastases, the trophic effect of pneumoperitoneum on malignant cells, and the surgical margins R0 [38]. In this meta-analysis, none of the 14 included studies identified port site metastases. Oncological of $\mathrm{R} 0$ resection is the gold standard of care of colorectal liver metastases. In this study, surgical margins R0 is assessed with no significant difference in laparoscopic liver resection compared with open liver resection. Instead, laparoscopic liver resection is prone to a lower recurrence rate than open liver resection. More importantly, the pooled analysis shows that 5-year disease-free survival and overall survival are not significant difference between laparoscopic and open liver resection. Therefore, laparoscopic liver resection has no compromises effect on oncologic outcomes compared with open liver resection for colorectal liver metastases.

There are several limitations in this meta-analysis. First, the included studies were mainly retrospective comparative studies with inherent limitations related to the significant risk of selection [39] and reporting bias [40].
The overall quality of evidence for all pooled results was relatively low. Second, colorectal liver metastases patients treated with laparoscopic liver resections were a highly selected population. Laparoscopic liver resection requires extensive experience and expertise than open liver resection and is associated with a learning curve. Finally, most of included studies were relatively small sample sizes and lack of long-term oncological data. Only 6 studies had follow-up longer than 5 years. Additionally, many of the studies were from single a single site, which may affect generalizability. Larger and well definitive randomized controlled trials would increase this study's predictive strength.

\section{MATERIALS AND METHODS}

\section{Study selection and search strategy}

A comprehensive search was performed in MEDLINE (PubMed), EMBASE and CENTRAL databases to identify all relevant studies available from their inception to October 18th 2016. We also searched trial registries of ongoing trials. When the criteria for inclusion or exclusion of a study were controversial, the corresponding author was consulted.

The search strategy followed the identification and screening guidelines established by PRISMA statement. The following Mesh search headings and key words were used: ("laparoscopic liver resection" or "laparoscopic hepatectomy") and ("colorectal cancer" or "colorectal liver metastases"). These terms were used in different Boolean combinations. Our search was restricted to full-length

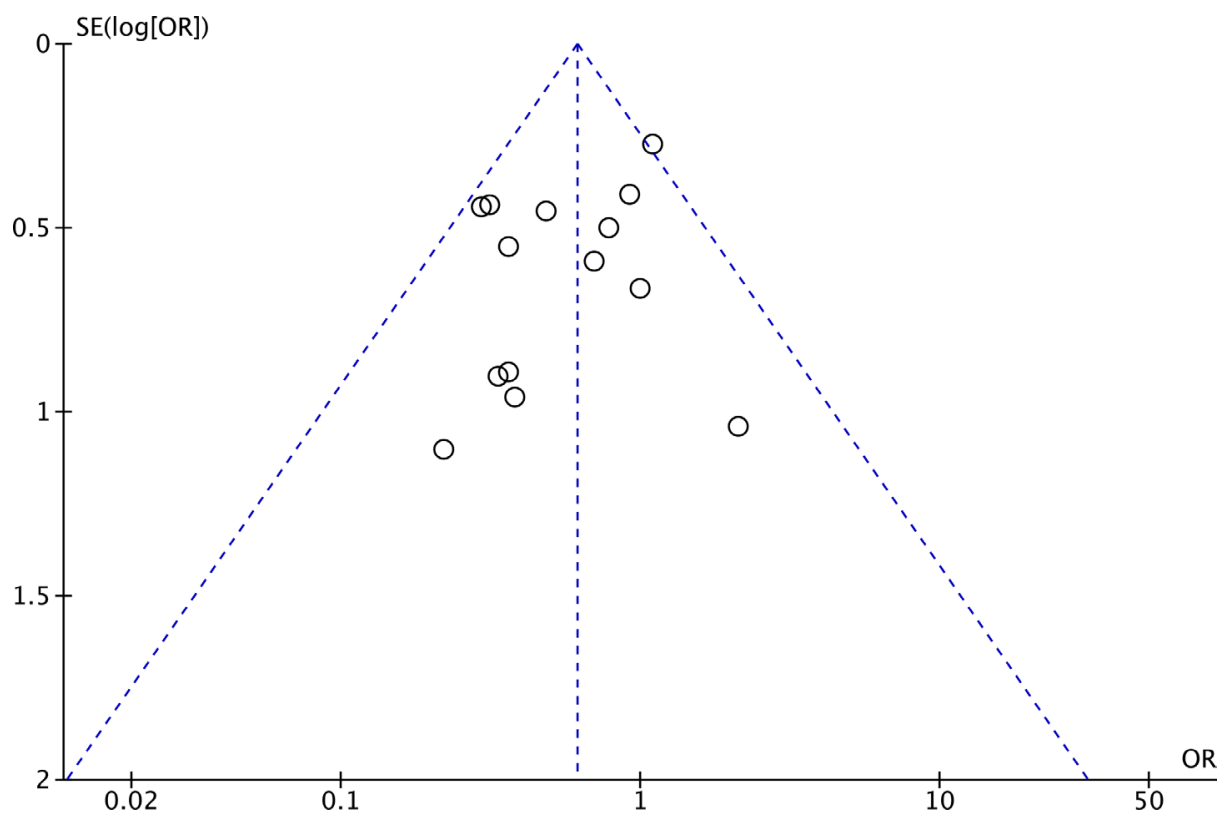

Figure 12: Funnel plot of postoperative complication morbidity analysis of publication bias. 
articles published in English. We retrieved all eligible studies and evaluated the reference lists of the identified studies and reviews.

\section{Inclusion criteria}

We included the following studies from the metaanalysis: (1) study design: comparing laparoscopic with open liver resection for colorectal liver metastases patients, (2) include more than 10 patients in each group (minimum of 20 patients), (3) the studies provided surgical and oncologic outcomes, and (4) available data for each surgical regimen. The most recent was used if dual (or multiple) studies were reported by the same institution. Study designs included randomized controlled trials and retrospective/prospective cohort or case-control studies.

\section{Exclusion criteria}

The exclusion criteria of the meta-analysis were the following: (1) studies not reporting clinical outcomes of effectiveness, (2) just one surgical regimen (laparoscopic or open) was reported, (3) other minimally invasive surgery such as radiofrequency ablation was compared, and (4) abstracts, letters, editorials and expert opinions, reviews without original data, case reports.

\section{Data extraction and outcomes of interest}

Data was independently extracted and the quality of included studies were screened by two review authors (T.Z. and X.S.). The two authors extracted the interest variable data of included studies and entered into a dedicated database. The following data of each study was extracted: study characteristics (first author, study designs, surgical approaches, numbers of patients, and follow-up time), demographic measures (publication year, country, age, and gender), surgical outcomes (operation time, blood loss, perioperative blood transfusion, postoperative complication morbidity, postoperative mortality, and hospitalization time), and oncologic outcomes (surgical margins R0, recurrence, overall survival, diseasefree survival). We defined perioperative mortality as 90-day hospital death, and hospitalization time including postoperative time and total hospital time. Local recurrence which were observed till the end of followup included hepatic only, extra-hepatic only, and both hepatic and extra-hepatic. Hepatic only and extra-hepatic complications were both contained in complications. The data accuracy and completeness were checked by two other authors (J.H. and L.W.). Discrepancies were resolved by consensus in all authors.

\section{Risk of bias assessment}

The risk of bias of the cohort studies was assessed using the Newcastle-Ottawa Scale [31]. The Newcastle-
Ottawa Scale star system ranged 0 to 9 stars: 4 stars for selection, 2 stars for comparability, and 3 stars for outcome. In this mate-analysis, a study of the final score $>6$ star was regard as a high-quality study. Risk of bias of the included studies were independently assessed by two review authors (T.Z. and X.S.). Disagreement was resolved by consensus in all authors.

\section{Data synthesis and statistical analysis}

This study was statistical analyzed by using Review Manager version 5.3.5 [41]. Most of included studies are retrospective cohort studies. So, OR is used to calculate the dichotomous data in this meta-analysis. The continuous data were calculated by MD with $95 \%$ CI. We derived the missing standard deviations from other statistics, such as $P$ values or $C I$ if needed. For example, $P=0.00001$ was assumed when a $P$ value was reported as $P<0.00001$. Cochran's $Q$ test and the degree of inconsistency $\left(I^{2}\right)$ were used to assess heterogeneity among combined study results. A fixed-effects model was used if a $P>0.05$ and $I^{2}<50 \%$. Otherwise, data were pooled by using the random-effects. $P<0.05$ indicated statistical significance in the integration results. Publication bias in outcomes was assessed and treated using standard methodology. The funnel plots were used to analyze publication bias.

\section{CONCLUSIONS}

Our meta-analysis summarizes the best available evidence for surgical and oncological results in directly comparative research studies of laparoscopic compared with open liver resection for colorectal liver metastases. This study demonstrates that laparoscopic liver resection is associated with lower blood loss, less requiring blood transfusion, lower postoperative complication morbidity, shorter hospitalization time, and lower recurrence rate. These findings provide evidence to support laparoscopic liver resection is efficacious for colorectal liver metastases. However, results are limited to none of randomized controlled trials and lack data about the longterm oncological results. The evidences call for larger, well design, and long-term outcomes studies to fully characterize the efficacy of laparoscopic liver resections for colorectal liver metastases.

\section{ACKNOWLEDGMENTS AND FUNDING}

This project was supported by National Natural Science Foundation of China (Grant No. 81630070, 81372312, 81472737, 81372330, 81572444, 81401308, 81402026, 81402454, 81502417, 81502543); Special Funds for National Key Sci-Tech Special Project of China (Grant No. 2016ZX10002019-005-002); Shanghai Science and Technology Committee (Grant No. 14ZR1409200, 15PJ1410600, 14ZD1900403, 16YF1415000,16ZR1400200); Shanghai Municipal 
Education Commission (Grant No. 14ZZ086); Science Fund for Creative Research Groups, NSFC, China (Grant NO. 81521091).

\section{CONFLICTS OF INTEREST}

The authors have declared that no competing interests exist.

\section{REFERENCES}

1. Jemal A, Bray F, Center MM, Ferlay J, Ward E, Forman D. Global cancer statistics. CA Cancer J Clin. 2011; 61:69-90.

2. Wang L, Sun Y, Zhao B, Zhang H, Yu Q, Yuan X. Chemotherapy plus targeted drugs in conversion therapy for potentially resectable colorectal liver metastases: a metaanalysis. Oncotarget. 2016; 7:55732-55740. doi: 10.18632/ oncotarget.9675.

3. Rees M, Tekkis PP, Welsh FK, O'Rourke T, John TG. Evaluation of long-term survival after hepatic resection for metastatic colorectal cancer: a multifactorial model of 929 patients. Ann Surg. 2008; 247:125-135.

4. Curley SA. Outcomes after surgical treatment of colorectal cancer liver metastases. Semin Oncol. 2005; 32:S109-111.

5. Pawlik TM, Scoggins CR, Zorzi D, Abdalla EK, Andres A, Eng C, Curley SA, Loyer EM, Muratore A, Mentha G, Capussotti L, Vauthey JN. Effect of surgical margin status on survival and site of recurrence after hepatic resection for colorectal metastases. Ann Surg. 2005; 241:715-722, discussion 722-714.

6. Fernandez FG, Drebin JA, Linehan DC, Dehdashti F, Siegel BA, Strasberg SM. Five-year survival after resection of hepatic metastases from colorectal cancer in patients screened by positron emission tomography with F-18 fluorodeoxyglucose (FDG-PET). Ann Surg. 2004; 240:438-447; discussion 447-450.

7. Zhou HS, Su XF, Fu XL, Wu GZ, Luo KL, Fang Z, Yu F, Liu H, Hu HJ, Chen LS, Cai B, Tian ZQ. Mesenchymal stem cells promote pancreatic adenocarcinoma cells invasion by transforming growth factor-betal induced epithelial-mesenchymal transition. Oncotarget. 2016; 7:41294-41305. doi: 10.18632/oncotarget.9319.

8. Mala T, Edwin B, Gladhaug I, Fosse E, Soreide O, Bergan A, Mathisen O. A comparative study of the short-term outcome following open and laparoscopic liver resection of colorectal metastases. Surg Endosc. 2002; 16:1059-1063.

9. Carswell KA, Sagias FG, Murgatroyd B, Rela M, Heaton N, Patel AG. Laparoscopic versus open left lateral segmentectomy. BMC Surg. 2009; 9:14.

10. Abu Hilal M, Di Fabio F, Teng MJ, Lykoudis P, Primrose JN, Pearce NW. Single-centre comparative study of laparoscopic versus open right hepatectomy. J Gastrointest Surg. 2011; 15:818-823.

11. Lee KF, Chong CN, Wong J, Cheung YS, Wong J, Lai P. Long-term results of laparoscopic hepatectomy versus open hepatectomy for hepatocellular carcinoma: a case-matched analysis. World J Surg. 2011; 35:2268-2274.
12. Fretland AA, Kazaryan AM, Bjornbeth BA, Flatmark K, Andersen MH, Tonnessen TI, Bjornelv GM, Fagerland MW, Kristiansen R, Oyri K, Edwin B. Open versus laparoscopic liver resection for colorectal liver metastases (the OsloCoMet Study): study protocol for a randomized controlled trial. Trials. 2015; 16:73.

13. Simillis C, Constantinides VA, Tekkis PP, Darzi A, Lovegrove R, Jiao L, Antoniou A. Laparoscopic versus open hepatic resections for benign and malignant neoplasms - a meta-analysis. Surgery. 2007; 141:203-211.

14. Yin Z, Fan X, Ye H, Yin D, Wang J. Short- and long-term outcomes after laparoscopic and open hepatectomy for hepatocellular carcinoma: a global systematic review and meta-analysis. Ann Surg Oncol. 2013; 20:1203-1215.

15. Zhou Y, Xiao Y, Wu L, Li B, Li H. Laparoscopic liver resection as a safe and efficacious alternative to open resection for colorectal liver metastasis: a meta-analysis. BMC Surg. 2013; 13:44.

16. Moher D, Liberati A, Tetzlaff J, Altman DG, Group P. Preferred reporting items for systematic reviews and meta-analyses: the PRISMA statement. PLoS Med. 2009; 6:e1000097.

17. Abu Hilal M, Underwood T, Zuccaro M, Primrose J, Pearce N. Short- and medium-term results of totally laparoscopic resection for colorectal liver metastases. Br J Surg. 2010; 97:927-933.

18. Beppu T, Wakabayashi G, Hasegawa K, Gotohda N, Mizuguchi T, Takahashi Y, Hirokawa F, Taniai N, Watanabe M, Katou M, Nagano H, Honda G, Baba H, et al. Long-term and perioperative outcomes of laparoscopic versus open liver resection for colorectal liver metastases with propensity score matching: a multi-institutional Japanese study. J Hepatobiliary Pancreat Sci. 2015; 22:711-720.

19. Castaing D, Vibert E, Ricca L, Azoulay D, Adam R, Gayet B. Oncologic Results of Laparoscopic Versus Open Hepatectomy for Colorectal Liver Metastases in Two Specialized Centers. Ann Surg. 2009; 250:849-855.

20. Cheung TT, Poon RTP, Yuen WK, Chok KSH, Tsang SHY, Yau T, Chan SC, Lo CM. Outcome of laparoscopic versus open hepatectomy for colorectal liver metastases. ANZ J Surg. 2012; 83:847-852.

21. de\&apos;Angelis N, Eshkenazy R, Brunetti F, Valente R, Costa M, Disabato M, Salloum C, Compagnon P, Laurent A, Azoulay D. Laparoscopic Versus Open Resection for Colorectal Liver Metastases: A Single-Center Study with Propensity Score Analysis. J Laparoendosc Adv Surg Tech A. $2015 ; 25: 12-20$.

22. Guerron AD, Aliyev S, Agcaoglu O, Aksoy E, Taskin HE, Aucejo F, Miller C, Fung J, Berber E. Laparoscopic versus open resection of colorectal liver metastasis. Surg Endosc. 2012; 27:1138-1143.

23. Inoue Y, Hayashi M, Tanaka R, Komeda K, Hirokawa F, Uchiyama K. Short-term results of laparoscopic versus open liver resection for liver metastasis from colorectal cancer: a comparative study. Am Surg. 2013; 79:495-501. 
24. Iwahashi S, Shimada M, Utsunomiya T, Imura S, Morine $\mathrm{Y}$, Ikemoto T, Arakawa Y, Mori H, Kanamoto M, Yamada S. Laparoscopic hepatic resection for metastatic liver tumor of colorectal cancer: comparative analysis of short- and longterm results. Surg Endosc. 2013; 28:80-84.

25. Kubota Y, Otsuka Y, Tsuchiya M, Katagiri T, Ishii J, Maeda T, Tamura A, Kaneko H. Efficacy of laparoscopic liver resection in colorectal liver metastases and the influence of preoperative chemotherapy. World J Surg Oncol. 2014; 12:351.

26. Mala T, Edwin B, Gladhaug I, Fosse E, Søreide O, Bergan A, Mathisen $\varnothing$. A comparative study of the shortterm outcome following open and laparoscopic liver resection of colorectal metastases. Surg Endosc. 2002; 16:1059-1063.

27. MD RMC, MBA CRSM, MD GGC, PhD KMMM and PhD RCGMIM. Laparoscopic versus open resection of hepatic colorectal metastases. Surgery. 2012; 152:567-574.

28. MD YH, MD HN, MD AS, MD TT, MD HI, MD HK, MD KO, PhD SNM and FACS GWMP. Long-term outcomes of laparoscopic versus open liver resection for liver metastases from colorectal cancer: A comparative analysis of 168 consecutive cases at a single center. Surgery. 2015; 157:1065-1072.

29. Qiu J, Chen S, Pankaj P, Wu H. Laparoscopic Hepatectomy for Hepatic Colorectal Metastases - A Retrospective Comparative Cohort Analysis and Literature Review. PLoS ONE. 2013; 8:e60153-60156.

30. Topal H, Tiek J, Aerts R, Topal B. Outcome of laparoscopic major liver resection for colorectal metastases. Surg Endosc. 2012; 26:2451-2455.

31. Stang A. Critical evaluation of the Newcastle-Ottawa scale for the assessment of the quality of nonrandomized studies in meta-analyses. Eur J Epidemiol. 2010; 25:603-605.

32. Higgins JP, Altman DG, Gotzsche PC, Juni P, Moher D, Oxman AD, Savovic J, Schulz KF, Weeks L, Sterne JA, Cochrane Bias Methods G, Cochrane Statistical Methods G. The Cochrane Collaboration's tool for assessing risk of bias in randomised trials. BMJ. 2011; 343:d5928.
33. Buell JF, Gayet B, Han HS, Wakabayashi G, Kim KH, Belli G, Cannon R, Saggi B, Keneko H, Koffron A, Brock G, Dagher I. Evaluation of stapler hepatectomy during a laparoscopic liver resection. HPB (Oxford). 2013; $15: 845-850$.

34. Nguyen KT, Laurent A, Dagher I, Geller DA, Steel J, Thomas MT, Marvin M, Ravindra KV, Mejia A, Lainas P, Franco D, Cherqui D, Buell JF, et al. Minimally invasive liver resection for metastatic colorectal cancer: a multiinstitutional, international report of safety, feasibility, and early outcomes. Ann Surg. 2009; 250:842-848.

35. Topal B, Tiek J, Fieuws S, Aerts R, Van Cutsem E, Roskams T, Prenen H. Minimally invasive liver surgery for metastases from colorectal cancer: oncologic outcome and prognostic factors. Surg Endosc. 2012; 26:2288-2298.

36. Jones C, Kelliher L, Dickinson M, Riga A, Worthington T, Scott MJ, Vandrevala T, Fry CH, Karanjia N, Quiney N. Randomized clinical trial on enhanced recovery versus standard care following open liver resection. Br J Surg. 2013; 100:1015-1024.

37. Abu Hilal M, McPhail MJ, Zeidan B, Zeidan S, Hallam MJ, Armstrong T, Primrose JN, Pearce NW. Laparoscopic versus open left lateral hepatic sectionectomy: A comparative study. Eur J Surg Oncol. 2008; 34:1285-1288.

38. Gutt CN, Riemer V, Kim ZG, Erceg J, Lorenz M. Impact of laparoscopic surgery on experimental hepatic metastases. Br J Surg. 2001; 88:371-375.

39. Lonjon G, Boutron I, Trinquart L, Ahmad N, Aim F, Nizard R, Ravaud P. Comparison of treatment effect estimates from prospective nonrandomized studies with propensity score analysis and randomized controlled trials of surgical procedures. Ann Surg. 2014; 259:18-25.

40. Clavien PA, Puhan MA. Biased reporting in surgery. Br J Surg. 2014; 101:591-592.

41. Higgins JPT GS. Cochrane Handbook for Systematic Reviews of Interventions Version.: The Cochrane Collaboration). 2011. 